\title{
A Cousin Formulation for Overlapped Domain Decomposition Applied to the Poisson Equation
}

\author{
Drazen Fabris ${ }^{1}$ and Sergio Zarantonello ${ }^{2}$ \\ 1 Santa Clara University, School of Engineering, Department of Mechanical \\ Engineering, 500 El Camino Real, Santa Clara, CA 95053, USA. \\ dfabris@scu.edu \\ 2 Department of Applied Mathematics, Santa Clara University and 3DGeo \\ Development Inc., 4633 Old Ironsides Drive, Santa Clara, CA 95054 \\ szarantonello@scu.edu
}

In this paper we present a domain decomposition method for the solution of a linear equation as a direct two-step procedure (in contrast to iterativebased Schwarz and Aitken-Schwarz procedures): first, local solutions to the nonhomogeneous equation are calculated on overlapping domains, and second, connection functions, solutions to the homogeneous equation, are calculated which satisfy the Cousin problem and correct for the initial solution mismatch at interior boundaries. The procedure is applied to Poisson's equation as a sample linear equation. The calculation of the connection functions is achieved through a classical orthogonal decomposition of the solution to Laplace's equation and can be achieved through progressive solutions in each direction for separable boundary conditions. The procedure is also applicable to more complicated domains with non-separable boundary conditions. A few examples will be given. The connection functions can be calculated to high precision and require minimal overlap of the subdomains.

\section{Introduction}

In the classical Schwarz procedure applied to a domain decomposition with overlapping subdomains the final solution is achieved through an iterative procedure updating the boundary conditions that are internal to the subdomains [Sch70] (see also optimized variants [GHN01]). The classical Schwarz technique is limited since the number of iterations scales geometrically with the number of subdomains and the rate of convergence depends strongly on the overlap. Recent work has shown that each successive iteration can be represented as a linear operator on the solution and that the final solution can be determined 
from the extrapolation of the linear convergence rate. This Aitken-Schwarz procedure has been extended to non-linear problems as a Steffensen-Schwarz procedure [BGO03, GT02a, GT02b]. In this paper we consider Poisson's equation in overlapped regular and irregular geometries as a model system for future applications to the Navier-Stokes equations. The matching requirement is based on equating the two local solutions functionally in the overlap region, a stricter requirement than the Schwarz procedure. This formulation leads to a Cousin like problem [GR65] and, for this linear problem, results in a constrained system for coupled solutions of the homogeneous form of the given equation.

First, we require a local Poisson solve on each subdomain. The local solutions in the overlapping regions pose a Cousin problem, and the difference in the values of the two functions satisfies the homogeneous form of the equation. Second, connection functions are calculated that satisfy the Cousin problem and smoothly patch the local solutions together. The connection functions are determined based on boundary information. This technique is similar to the superposition, filtering, and patching technique of Israeli et al. [IVA93] but uses an exact representation of the connection functions and results in a fully coupled system. In two dimensions, for rectangular computational domains, each connection function can be decomposed into the sum of four separate connection functions. In each direction the solutions are coupled at the local boundaries and lead to a set of simultaneous equations that can be solved for the coefficients. Solutions in multiple dimensions can be achieved by recursively treating the connection functions in each dimension. Hence a three-dimensional solution requires three applications of the procedure.

\section{Definitions, Cousin Problem, and Local Solutions}

Let $\Omega$ be the domain of interest. Assume that the domain can be decomposed into a finite set of open coverings $\Omega_{i}$ such that each domain has a finite overlap with its neighboring domains. In particular, we consider rectangular and L-shaped domains.

We seek to solve the linear Poisson equation as a model problem $\Delta p=f$ in $\Omega$ subject to $B p=b$ on $\partial \Omega$, with Dirichlet boundary values. We assume $f \in C(\bar{\Omega})$ and $b \in C(\partial \Omega)$ for $n \geq 2$.

First, the local Poisson problem is solved

$$
\Delta q_{i}=f \quad \text { in } \quad \Omega_{i} \text { with } B q_{i}=\tilde{b}_{i} \quad \text { on } \partial \Omega_{i}
$$

and second, a set of connection functions are generated

$$
\Delta h_{i}=0 \quad \text { in } \Omega_{i} \text { with } B h_{i}=\tilde{c_{i}} \text { on } \partial \Omega_{i}
$$

where $h_{i}$ satisfies a Cousin problem and is determined by boundary values. Here $\tilde{b}_{i}$ is a continuous non-unique extension of the $b$ on $\partial \Omega_{i}$. The $\tilde{c}$ 's are 
determined by the mismatch in the local solutions as will be discussed in section 3 .

Cousin Problem: Suppose, for every pair of subdomains $\Omega_{\alpha} \cap \Omega_{\beta} \neq \emptyset$, we have $h_{\alpha \beta}$ such that $\Delta h_{\alpha \beta}=0$ and $h_{\alpha \beta}=0$ on $\left(\partial \Omega_{\alpha} \cap \partial \Omega_{\beta}\right)$ so that the following cocycle properties are satisfied:

$$
h_{\alpha \alpha}=0 \quad h_{\alpha \beta}=-h_{\beta \alpha} \quad h_{\alpha \beta}+h_{\beta \gamma}=h_{\alpha \gamma}
$$

The additive Cousin Problem with data $\left\{h_{\alpha \beta}\right\}$ is the problem of finding functions $h_{\alpha}$ such that $\Delta h_{\alpha}=0$ in $\Omega_{\alpha}$ and $h_{\alpha \beta}=h_{\beta}-h_{\alpha}$ in $\bar{\Omega}_{\alpha} \cap \bar{\Omega}_{\beta}$ with homogeneous Dirichlet boundary conditions on the boundary of $\partial \bar{\Omega}_{\alpha}$ not in $\partial \Omega_{\alpha} \cap \partial \Omega_{\beta}$ [GR65].

If $\left\{q_{\alpha}\right\}$ is a complete set of exact solutions to the subdomain problems (1), then the functions generated from the difference of local solutions,

$$
h_{\alpha \beta}=q_{\alpha}-q_{\beta},
$$

satisfy $\Delta h_{\alpha \beta}=0$, and the cocycle properties (3) are trivially verified. Once the functions $\left\{h_{\alpha}\right\}$ are found, by rearranging

$$
q_{\alpha}-q_{\beta}=h_{\alpha \beta}=h_{\beta}-h_{\alpha}
$$

we get $p_{\alpha}=q_{\alpha}+h_{\alpha}=p_{\beta}=q_{\beta}+h_{\beta}$ in $\bar{\Omega}_{\alpha} \cap \bar{\Omega}_{\beta}$ and the global solution becomes $p=\bigcup p_{\alpha}$ in $\bar{\Omega}$. The key to the procedure is the computational simplicity of the computation of the homogeneous solutions and the superposition of the local solutions and connection functions.

Generation of accurate local solutions is critical to overall accuracy of the technique and may limit the final order of accuracy. We use two methods for calculating the local solutions: first, a direction solution via a Schur decomposition [Can88], and second a pseudo-spectral Fourier method in combination with a boundary regularization procedure to subtract aperiodicity in the boundary conditions [ZFC]. The local solutions can be difficult to compute in that they arise from local problems that are nonperiodic and are subject to the interpolated boundary conditions. A range of spectral methods has been developed to approach this problem [AIV98, Sko75].

In the first method the solution is determined by the finite difference expansion and, in this case, results in a second order truncation error and a second order accurate solution. The second method is outlined in Zarantonello, Fabris, and Chiappari $[\mathrm{ZFC}]$. In this approach the right hand side is preconditioned by removing aperiodic behavior in the harmonic extension of $f$. This technique has been introduced by Sköllermo [Sko75] and implemented by Averbuch et al. [AIV98].

\section{Examples and Results}

Let $\left\{\Omega_{n}\right\}$ be a finite set of open coverings of $\Omega$, a rectangular domain $(a, b) \times$ $(c, d)=\left\{(x, y) \in \mathbf{R}^{2} \mid a<x<b, c<y<d\right\}$, such that $\Omega_{n}=\left(a_{n}, b_{n}\right) \times(c, d)$ 
and $a_{n}<b_{n-1}<a_{n+1}<b_{n}$. We refer to this set as a rectangular domain decomposition of $\Omega$ and note that the domains have a finite overlap and are horizontally aligned, Figure 1 . This decomposition can be extended into a two or three dimensional array of overlapping domains in a similar manner.

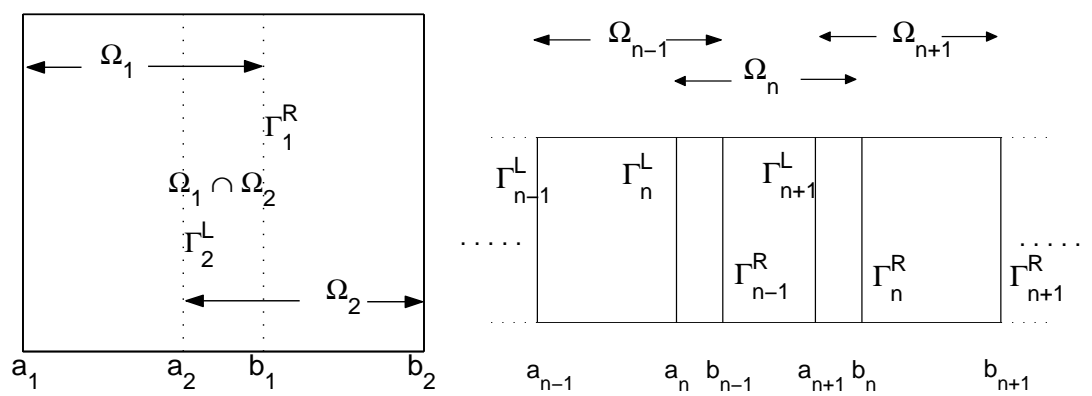

Fig. 1. Two subdomains and a row of subdomains.

With two overlapping domains consider $\Gamma_{2}^{L}$ and $\Gamma_{1}^{R}$ to be the interior boundaries of $\Omega_{2}$ and $\Omega_{1}$ as shown in Figure 1. The Cousin data consists of the single function $h_{12}=q_{1}-q_{2}$ defined on $\Omega_{1} \cap \Omega_{2}$. The connection functions, $h_{1}$ and $h_{2}$, are solutions to $\Delta h=0$ and can be generated from boundary conditions expressed in an orthogonal basis appropriate to the specific domain, in this case an expansion in sines and hyperbolic sines. Since the initial local solutions, $q_{\alpha}$, satisfy homogeneous global boundary conditions that are extended to homogeneous boundary conditions on $\Gamma_{2}^{L}$ and $\Gamma_{1}^{R}$, the connection functions each have only one non-homogeneous boundary conditions. For the purpose of illustration

$$
h_{1}(x, y)=\sum_{k=1}^{\infty} R_{k} \frac{\sinh k \pi\left(x-a_{1}\right)}{\sinh k \pi\left(b_{1}-a_{1}\right)} \sin k \pi y
$$

and

$$
h_{2}(x, y)=\sum_{k=1}^{\infty} L_{k} \frac{\sinh k \pi\left(b_{2}-x\right)}{\sinh k \pi\left(b_{2}-a_{2}\right)} \sin k \pi y
$$

in the rectangular domain. Here $\left\{L_{k}\right\}$ and $\left\{R_{k}\right\}$ are the coefficients derived from the sine expansions of the boundary data on the left and right edges of each rectangular domain. Now, equation 5 is used to identify the boundary data

$$
\left.q_{1}\right|_{\Gamma_{2}^{L}}-\left.q_{2}\right|_{\Gamma_{2}^{L}}=\left.h_{12}\right|_{\Gamma_{2}^{L}}=\left.h_{2}\right|_{\Gamma_{2}^{L}}-\left.h_{1}\right|_{\Gamma_{2}^{L}}
$$

and

$$
\left.q_{2}\right|_{\Gamma_{1}^{R}}-\left.q_{1}\right|_{\Gamma_{1}^{R}}=\left.h_{12}\right|_{\Gamma_{1}^{R}}=\left.h_{1}\right|_{\Gamma_{1}^{R}}-\left.h_{2}\right|_{\Gamma_{1}^{R}}
$$

Using the data from $q_{1}$ and $q_{2}$, we expand $h_{12}$ on the same sine basis as (6) and $(7)$ 


$$
h_{12}(x, y)=\sum_{k=1}^{\infty}\left[T_{k} \frac{\sinh k \pi\left(x-a_{2}\right)}{\sinh k \pi\left(b_{1}-a_{2}\right)}-S_{k} \frac{\sinh k \pi\left(b_{1}-x\right)}{\sinh k \pi\left(b_{1}-a_{2}\right)}\right] \sin k \pi y .
$$

Using the eigenexpansions in (6), (7), and (10), equations (8) and (9) decouple in each wave number and can be solved simultaneously for each pair of expansion coefficients, $L_{k}$ and $R_{k}$, independently.

In the more general case of multiple domains, $\Omega_{n}$, connected in a row with only overlap of two adjacent domains the simultaneous system reduces to

$$
\left[\begin{array}{ccccccc}
D_{1, k} & 1 & 0 & 0 & 0 & \ldots & 0 \\
1 & A_{2, k} & B_{2, k} & 0 & 0 & \ldots & 0 \\
0 & C_{2, k} & D_{2, k} & 1 & 0 & \ldots & 0 \\
0 & 0 & 1 & A_{3, k} & B_{3, k} & . & 0 \\
0 & 0 & 0 & C_{3, k} & D_{3, k} & . & 0 \\
. & . & . & . & . & . & . \\
0 & 0 & 0 & . & . & . & A_{n, k}
\end{array}\right]\left[\begin{array}{c}
R_{1, k} \\
L_{2, k} \\
R_{2, k} \\
L_{3, k} \\
R_{3, k} \\
\ldots \\
L_{n, k}
\end{array}\right]=\left[\begin{array}{c}
S_{1, k} \\
T_{2, k} \\
S_{2, k} \\
T_{3, k} \\
S_{3, k} \\
\ldots \\
T_{n, k},
\end{array}\right]
$$

where

$$
\begin{array}{cc}
A_{n, k}=-\frac{\sinh k \pi\left(b_{n}-b_{n-1}\right)}{\sinh k \pi\left(b_{n}-a_{n}\right)}, & D_{n, k}=-\frac{\sinh k \pi\left(a_{n+1}-a_{n}\right)}{\sinh k \pi\left(b_{n}-a_{n}\right)}, \\
B_{n, k}=-\frac{\sinh k \pi\left(b_{n-1}-a_{n}\right)}{\sinh k \pi\left(b_{n}-a_{n}\right)}, & C_{n, k}=-\frac{\sinh k \pi\left(b_{n}-a_{n+1}\right)}{\sinh k \pi\left(b_{n}-a_{n}\right)} .
\end{array}
$$

In this system each row is normalized by the hyperbolic sine factors in equation (10). In the internal subdomains, the subdomains that overlap with two other subdomains, the connection function contains both left and right coefficients.

Since the subdomains overlap, the determinant of (11) is strictly positive and uniformly bounded away from zero. The solution $\left\{h_{n}\right\}$ depends continuously on the data $\left\{h_{n, n-1}\right\}$, and the procedure for solving the Cousin Problem is stable. The elements, equation (12), of the matrix in equation (11) are determined purely by the nature of the overlap, and the connection function in any one subdomain depends only on the local solutions in all of the other subdomains, but not on the other connection functions, $R_{j, k}=f_{j}\left(\left\{S_{k}\right\},\left\{T_{k}\right\}\right)$ and $L_{j, k}=g_{j}\left(\left\{S_{k}\right\},\left\{T_{k}\right\}\right)$. In essence this reduces the iterative problem to a two-step direct solution.

In a computational framework using a finite expansion each function $h_{n, n-1}=q_{n}-q_{n-1}$ is defined on a collocation grid of dimensions $M_{x ; n, n-1} \times$ $M_{y}$, the sine series (6), (7), and (10) become sine polynomials of order $M_{y}$, the sine coefficients are calculated via a Fast Sine Transform, the equation coefficients (12) are precalculated, and (11) reduces to $M_{y}$ systems of tridiagonal equations in $2 N-2$ unknowns.

We consider two test cases to demonstrate the method. In [GT02a] Garbey and Tromeur-Dervout proposed the problem $f(x, y)=2 y(y-1)+2 x^{2}-$ 0.5 , with exact solution $p(x, y)=\left(x^{2}-0.25\right) y(y-1)$ defined on the unit square. This particular case allows an exact solution to be computed with a 
second order discretization. Results are given in Table 1. Our $L_{\infty}$ errors are comparable to those of Garbey and Tromeur-Dervout and are on the order of machine precision. In this case the truncation error is exactly zero for the $2^{\text {nd }}$ order technique.

Table 1. First case considered, $L_{2}$ and $L_{\infty}$ relative to the same norms for the solution.

\begin{tabular}{lllll}
\hline $\begin{array}{l}\text { total nodes } \\
\text { (subdomains) }\end{array}$ & $\begin{array}{l}\text { spectral } \\
\operatorname{err}_{L_{2}}\end{array}$ & $\begin{array}{l}2^{\text {nd }} \text { order } \\
\operatorname{err}_{L_{2}}\end{array}$ & $\begin{array}{l}2^{\text {nd }} \text { order } \\
\operatorname{err}_{L_{\infty}}\end{array}$ & $\begin{array}{l}\operatorname{err}_{L_{\infty}}, \text { Garbey \& } \\
\text { Tromeur-Dervout }\end{array}$ \\
\hline $66 \times 66(2 \times 2)$ & $7.8501 \mathrm{e}-10$ & $4.7668 \mathrm{e}-14$ & $4.3161 \mathrm{e}-14$ & $3.8589 \mathrm{e}-13$ \\
$66 \times 66(4 \times 4)$ & $4.2206 \mathrm{e}-9$ & $4.7668 \mathrm{e}-14$ & $4.9009 \mathrm{e}-14$ & $4.2577 \mathrm{e}-14$ \\
$66 \times 66(8 \times 8)$ & $1.8064 \mathrm{e}-8$ & $9.5740 \mathrm{e}-14$ & $6.0633 \mathrm{e}-14$ & $2.2204 \mathrm{e}-15$ \\
$66 \times 66(16 \times 16)$ & $7.2144 \mathrm{e}-8$ & $2.2889 \mathrm{e}-13$ & $1.1517 \mathrm{e}-13$ & $1.1380 \mathrm{e}-15$ \\
$258 \times 258(2 \times 2)$ & $3.0977 \mathrm{e}-12$ & $7.5506 \mathrm{e}-13$ & $5.6097 \mathrm{e}-13$ & $1.3513 \mathrm{e}-11$ \\
$258 \times 258(4 \times 4$ & $1.6964 \mathrm{e}-11$ & $1.1543 \mathrm{e}-12$ & $9.0958 \mathrm{e}-13$ & $2.8467 \mathrm{e}-12$ \\
$258 \times 258(8 \times 8)$ & $7.3314 \mathrm{e}-11$ & $1.4259 \mathrm{e}-12$ & $1.1116 \mathrm{e}-12$ & $1.3563 \mathrm{e}-12$ \\
$258 \times 258(16 \times 16)$ & $2.9883 \mathrm{e}-10$ & $9.0078 \mathrm{e}-13$ & $6.5830 \mathrm{e}-13$ & $8.4238 \mathrm{e}-14$ \\
\hline
\end{tabular}

Second, we consider $f(x, y)=6 e^{x+y} x y(-3+y+x+x y)$, with the exact solution $p(x, y)=3 e^{x+y} x y(1-x)(1-y)$. This is Problem 4.1 of Rice et al. [RHD81]. Results are shown in Table 2. It is a commonly used example of an analytic problem with homogeneous Dirichlet boundary values. Rice [RHD81] considers only a single subdomain setting. The spectral solution is more accurate due to a higher order approximation. Figure 2 shows the solution and error for the third case in Table 2.

Table 2. Second case considered. The spectral method uses a local solution that is fourth order accurate.

\begin{tabular}{llll}
\hline $\begin{array}{l}\text { total nodes } \\
(\text { subdomains })\end{array}$ & $\begin{array}{l}\text { spectral } \\
\operatorname{err}_{L_{2}}\end{array}$ & $\begin{array}{l}\text { spectral } \\
\operatorname{err}_{L_{\infty}}\end{array}$ & $\begin{array}{l}2^{\text {nd }} \text { order } \\
\operatorname{err}_{L_{2}}\end{array}$ \\
\hline $18 \times 18(2 \times 2)$ & $2.8047 \mathrm{e}-7$ & $1.3978 \mathrm{e}-6$ & $3.6947 \mathrm{e}-4$ \\
$34 \times 34(2 \times 2)$ & $1.0409 \mathrm{e}-8$ & $9.6288 \mathrm{e}-8$ & $8.2776 \mathrm{e}-5$ \\
$66 \times 66(2 \times 2)$ & $3.6829 \mathrm{e}-10$ & $6.3610 \mathrm{e}-9$ & $1.9237 \mathrm{e}-5$ \\
$130 \times 130(2 \times 2)$ & $1.2921 \mathrm{e}-11$ & $4.0955 \mathrm{e}-10$ & $4.6102 \mathrm{e}-6$ \\
$258 \times 258(2 \times 2)$ & $4.7057 \mathrm{e}-13$ & $2.5989 \mathrm{e}-11$ & $1.1266 \mathrm{e}-6$ \\
\hline
\end{tabular}

The results are given for two local solvers, the spectral and Schur decomposition. We note that the Schur decomposition provides the exact solution for the first problem since the truncation error is exactly zero. The results approach machine accuracy and are comparable with or better than the best 

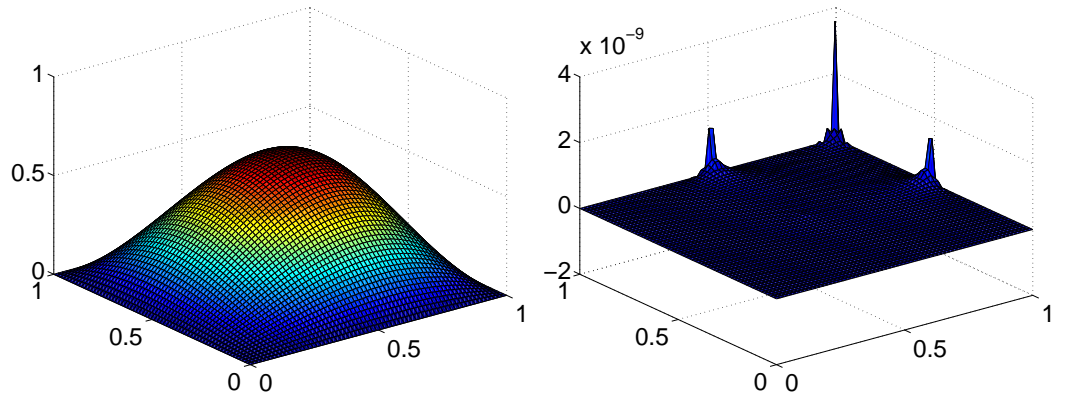

Fig. 2. Solution and error.

published results for this particular problem. Figure 3 shows the application of the procedure to an L-shaped domain.
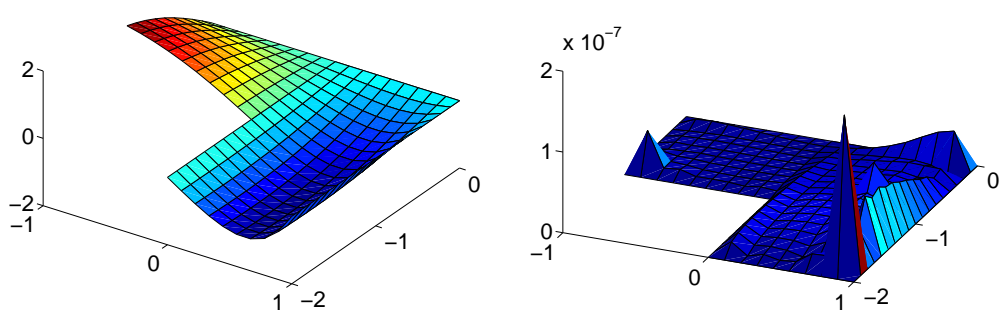

Fig. 3. Two rectangular domains overlapping into an L-shape: solution and error.

\section{Conclusions}

A direct method for solution of linear elliptic problems through domain decomposition has been presented as applied to the Poisson equation. The procedure calculates local solutions on each domain and computes connection functions as solutions to a Cousin problem that correct for the mismatch on the internal boundaries and overlap domains.

In regular rectangular decompositions, eigenfunction expansions for the connection boundary value problem can be calculated directly with each component separated. In two dimensions (or more) each direction needs to be calculated successively with a second Cousin problem generated after calculation of each connection function. In L-shaped and more complicated domains the connection functions can still be calculated but require full coupling of all the eigenfunctions. 
The final procedure is as accurate as the accelerated Schwarz procedure with the order of accuracy determined by the local solution procedure. The benefit of the procedure is the improvement in the calculation of the connection functions, directly through eigenexpansions, that is half as expensive as a second iteration of the Schwarz procedure. Matching the solution in the overlap provides a stronger condition than transmission of boundary data. Furthermore, the procedure allows for the computation of connection solutions in more complicated domains and successively in two or more dimensions.

\section{References}

[AIV98] Averbuch, A., Israeli, M., Vozovoi, L.: A fast Poisson solver of arbitrary order accuracy in rectangular regions. SIAM J. Sci. Comput., 19, No. 3, 933-952 (1998)

[BGO03] Baranger, J., Garbey, M., Oudin-Dardun, F.: On Aitken like acceleration of Schwarz domain decomposition method using generalized Fourier. In: Herrera, I., Keyes, D.E., Widlund, O.B., Yates, R. (ed) Proc. of the $14^{\text {th }}$ Int. Conf. on Domain Decomposition Methods. UNAM, Mexico City 341$348(2003)$

[Can88] Canuto, C., Hussaini, M.Y., Quarteroni, A., Zang, T.A.: Spectral methods in fluid dynamics. Springer-Verlag, Berlin (1988)

[GHN01] Gander, M.J., Halpern, L., Nataf, F.: Optimized Schwarz Methods. In: Chan, T., Kako, T., Kawarada, H., Pironneau, O. (ed) Proc. of the $12^{\text {th }}$ Int. Conf. on Domain Decomposition Methods. ddm.org, 15-27 (2001)

[GT02a] Garbey, M. Tromeur-Dervout, D.: Aitken-Schwarz algorithm on Cartesian grid. In: Debit, N., Garbey, M., Hoppe, R., Périaux, J., Keyes, D., Kuznetsov, Y. (ed) Proc. of the $13^{\text {th }}$ Int. Conf. on Domain Decomposition Methods. dd.org, 53-66 (2002)

[GT02b] Garbey, M, Tromeur-Dervout, D.: On some Aitken-like acceleration of the Schwarz method. Int. J. Numer. Meth. in Fluids, 40, 1493-1513 (2002)

[GR65] Gunning, R.C., Rossi, H.: Analytic functions of several complex variables. Prentice-Hall Series in Modern Analysis, Prentice-Hall Inc., Englewood Cliffs (1965)

[IVA93] Israeli, M., Vozovoi, L., Averbuch, A.: Spectral Multidomain Technique with Local Fourier Basis. Journal of Scientific Computing, 8, No. 2, 135149 (1993)

[RHD81] Rice, J.R., Houstis, E.N., Dyksen, W.R.: A population of linear, second order, elliptic partial differential equations on rectangular domains, part 1. Math. Comp., 36, 475-484 (1981)

[Sch70] Schwarz, H.A.: Ueber einen Grenzübergang durch alternierendes Verfahren. Vierteljahrsschrif der Naturforschenden Gesellschaft in Zürich, 15, 272-286 (1870)

[Sko75] Sköllermo, G.: A Fourier method for numerical solution of Poisson's equation. Math. Comp., 29 , 697-711 (1975)

[ZFC] Zarantonello, S., Fabris, D., Chiappari, S.: The use of a redundant basis for the spectral solution of Poisson's equation. in preparation 